Research Paper

\title{
Surgical Compliance and Outcomes in Gastric Cancer: a population-based cohort study
}

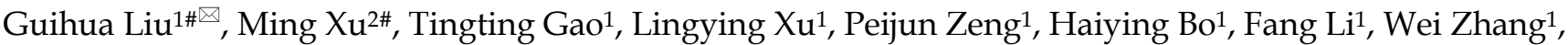 \\ Zhengting Wang ${ }^{3 凶}$ \\ 1. Department of General Practice, Zhongshan Street Community Health Center of Songjiang District, Shanghai, 201600, China \\ 2. Department of General Surgery, Tongren Hospital, School of Medicine, Shanghai Jiao Tong University, Shanghai, 200001, China \\ 3. Department of Gastroenterology, Ruijin Hospital, School of Medicine, Shanghai Jiao Tong University, Shanghai, 200001, China \\ \# Contributed equally \\ 凹 Corresponding author: Guihua Liu:liugh_xyqy@126.com, Zhengting Wang: zhengtingwang@shsmu.edu.cn \\ (c) Ivyspring International Publisher. This is an open access article distributed under the terms of the Creative Commons Attribution (CC BY-NC) license \\ (https://creativecommons.org/licenses/by-nc/4.0/). See http://ivyspring.com/terms for full terms and conditions.
}

Received: 2018.08.08; Accepted: 2019.01.09; Published: 2019.02.02

\begin{abstract}
Background: Surgical resection is one of curative treatment for gastric cancer (GC), however, a set of patients show poor surgical compliance in the USA. We aimed to identify the risk factors associated with surgical compliance and investigate the difference in survival.

Methods: GC patients diagnosed between 1973 and 2014 were identified from the Surveillance Epidemiology and End Results (SEER) databases. Based on different surgical compliance and treatment regimen, patients were classified into three subgroups: surgical compliance group, surgical noncompliance group, and non-surgical group. Multivariable Logistic regression analysis was adopted to identify the factors related to surgical compliance; Multivariable Cox regression was used to investigate the prognostic factors. Overall survival (OS) and cancer-specific survival (CSS) were analyzed using the Kaplan-Meier estimator method.

Results: Of 79374 GC patients who were recommended for surgical therapy, 15201(19.2\%) cases did not perform surgery. Poor compliance of surgery was related to old age, American Indian/Alaska Native race, poor grading/late staging, single/widowed status, lower socioeconomic status and earlier time of diagnosis. As expected, GC patients of surgical compliance group showed significantly more favorable survival than the other two groups $(\mathrm{P}<0.0001)$; notably, the outcome of surgical noncompliance group came close to that of non-surgical group.

Conclusion: GC patients of poor surgical compliance demonstrated adverse survival, which was comparable to that of non-surgical patients. The poor surgical compliance was associated with older age, American Indian/Alaska Native race, poor tissue differentiation/advanced stage of tumor, single/widowed status, lower socioeconomic status and earlier time of diagnosis.
\end{abstract}

Key words: Gastric cancer, Surgical Compliance, SEER, Survival, Outcome

\section{Introduction}

GC is one of the most common cancers worldwide, and it is the third leading cause of cancer death [1]. A total of more than 950000 new cases have been diagnosed every year, with about 720000 patients died from GC in 2012[2]. GC is more prevalence in men than women and has obvious ethnic and regional variations $[3,4]$. In recent years, the incidence of GC has gradually decreased in most parts of the world, but it remains great burden in Asia, central and eastern Europe and Latin America $[4,5]$.

Treatment for GC is mainly dependent on tumor stage. The only potentially curative treatment is surgical resection [6, 7], including endoscopic resection, minimally invasive surgery, or open gastrectomy etc $[8,9]$. For the late stage patients, 
surgery alone is not enough, due to the high rate of local recurrence or distant metastasis in GC [10]. Then, adjuvant therapies are needed to improve survival. Previous studies have demonstrated that chemotherapy or chemoradiotherapy combined with surgery can yield survival benefit comparing to surgery alone to some extent $[11,12]$. Early GC has a relatively favorable prognosis, however, because of no specific symptom in the early stage, a considerable proportion of gastric cancers are diagnosed in late stage [13]. Although great advances have been made in treatment, the prognosis of GC is still dismal; the global 5-year overall survival for gastric cancer patients is low in most areas in the world (25\% 30\%), and the outcomes are even worse when tumors are disseminated ( 10\%) [14, 15].

In addition to the high malignancy of GC itself, undertreatment in GC is considered to be an important reason for poor prognosis, which is closely associated with the therapeutic regimen and patients' compliance. Generally, the elderly GC patients are prone to have inadequate therapy. Several studies reveal that more preexisting comorbidities are found in elderly GC patients [16, 17], which may augment the difficulties and risks of surgical treatment [18]. Thus, gastrectomy with reduced nodal dissection is an alternative in order to reduce postoperative complications. However, surgical undertreatment is associated with worse survival among GC patients in a prospective randomized trial [19]. Notably, a set number of GC patients who are recommended for surgery have no surgical operation, but the reason is elusive. Surgical incompliance is one kind of serious undertreatment, which should be affected by multiple factors, including demographic characteristics and clinicopathological features etc. Based on this hypothesis, we aim to identify the covariates which contribute to surgical incompliance in this retrospective analysis. The identification of modifiable factors will contribute to the buildup of effective interventions; consequently, ensure the surgical compliance in GC and improve the outcome ultimately.

\section{Materials and methods}

The malignant GC patients in our study were retrieved from the Surveillance Epidemiology and End Results (SEER) database, which provides patients' demographic information and cancer characteristics for approximately $28 \%$ of the US population. All the malignant gastric cancers in our study have been confirmed through pathologic examination. Thus, we identified 116718 GC patients in the final cohort who were eligible for the study. All the cases of GC patients were retrieved via the
SEER ${ }^{*}$ Stat software (version 8.3.5). The exact database was Surveillance, Epidemiology, and End Results (SEER) Program (www.seer.cancer.gov) Research Data (1973-2014), National Cancer Institute, DCCPS, Surveillance Research Program, released April 2017, based on the November 2016 submission.

Our study was from the public data of SEER database, as no personal identifying information was used, the informed consent was waived.

\section{Statistical methods}

The diagnosis time spans 40 years in this SEER database, then patients were factiously classified into four groups based on the time of diagnosis in chronological order. The groups are as follows: Group A: 1973-1983, Group B: 1984-1993, Group C: 1994-2003, and Group D: 2004-2014. Based on different treatment regimens and patients' compliance, these patients were categorized into three subgroups. For ease of description, patients who accepted surgery were termed as surgical compliance group; the patients who were recommended for surgery but had no surgical operation were classified into surgical noncompliance group; the patients who were not recommended for surgery belonged to non-surgical group.

The patients' demographic and clinical characteristics were summarized and Chi-square test was introduced to compare the categorical variables among different groups. In order to probe the potential variables that were associated with patients' compliance to surgery, Multivariate Logistic regression analysis was adopted. The logistic model incorporated nine variables, which were the time of diagnosis, gender, age, race, ethnic origin, pathological grading, tumor stage, marital status, education level, and economic income.

The Multivariate Cox proportional regression analysis was applied to evaluate the hazard ratio (HR) and the $95 \%$ confident interval (CI) for the potential prognostic factors, including time of diagnosis, gender, age, pathological grading, tumor stage, race, ethnic origin, marital status, education level, economic income, and surgery status.

The Kaplan-Meier estimator method was applied to estimate survival in different groups, and log-rank tests were used for comparison. The overall survival (OS) time is from the date of diagnosis to death from any cause or the date on which data were censored. Cause-specific survival (CSS) is a net survival measure. In our study, CSS estimates the probability of surviving GC. The patients are censored who are still alive at the date of last follow-up or those who die from other causes except for GC. All statistical analyses were performed by $\mathrm{R}$ software 
(3.4.2 version). All P-values were two-sided in our analysis unless otherwise specified.

\section{Results}

\section{The demographic and clinicopathological characteristics.}

The cohort in our study included a total of 116718 GC patients. Among them, 79374 patients were recommended for surgical therapy and the rest ones (37344 cases) for non-surgical treatment; however, 15201 (19.2\%) out of 79374 patients did not perform surgery.

The relationship between clinicopathological features and surgical compliance was shown in Table 1. The compliance of surgery gradually improved over time; a significant proportion of patients who did not follow the surgery were diagnosed during the time of $1973-1983$, up to $39.2 \%$, and the percentage declined to $14.5 \%$ in the latest decade. The ratio of male to female was close between the two groups. Because there was no significant difference between them, this variable was filtered out when performing Multivariable logistic regression analysis.

Table 1. The baseline demographic and clinical characteristics for GC in our study

\begin{tabular}{|c|c|c|c|}
\hline & $\begin{array}{l}\text { Surgical } \\
\text { compliance } \\
\text { group } \\
\mathrm{N}=64173(80.8 \%)\end{array}$ & $\begin{array}{l}\text { Surgical } \\
\text { noncompliance } \\
\text { group } \\
\mathrm{N}=15201(19.2 \%)\end{array}$ & $\begin{array}{l}\text { Chi-square } \\
\text { d Test }\end{array}$ \\
\hline \multicolumn{4}{|l|}{ Diagnosis time } \\
\hline group A:1974-1983 & $9775(15.2 \%)$ & $5959(39.2 \%)$ & $<0.0001$ \\
\hline group B:1984-1993 & $10613(16.5 \%)$ & $3342(22 \%)$ & \\
\hline group C:1994-2003 & $18473(28.8 \%)$ & $3700(24.3 \%)$ & \\
\hline group D:2004-2014 & $25312(39.4 \%)$ & $2200(14.5 \%)$ & \\
\hline \multicolumn{4}{|l|}{ Gender } \\
\hline Female & $24929(38.8 \%)$ & $5843(38.4 \%)$ & 0.3577 \\
\hline Male & $39244(61.2 \%)$ & $9358(61.6 \%)$ & \\
\hline \multicolumn{4}{|l|}{ Age } \\
\hline$<50 y$ & $7966(12.4 \%)$ & $1202(7.9 \%)$ & $<0.0001$ \\
\hline $50-64 y$ & $19083(29.7 \%)$ & $3458(22.7 \%)$ & \\
\hline $65-79 y$ & $27362(42.6 \%)$ & $6037(39.7 \%)$ & \\
\hline$\geq 80 y$ & $9762(15.2 \%)$ & $4504(29.6 \%)$ & \\
\hline \multicolumn{4}{|l|}{ Race } \\
\hline White & $44921(70 \%)$ & $11500(75.7 \%)$ & $<0.0001$ \\
\hline $\begin{array}{l}\text { American } \\
\text { Indian/Alaska Native }\end{array}$ & $444(0.7 \%)$ & $156(1.0 \%)$ & \\
\hline Asian or Pacific Islander & $10802(16.8 \%)$ & $1576(10.4 \%)$ & \\
\hline Black & $8006(12.5 \%)$ & $1969(13 \%)$ & \\
\hline \multicolumn{4}{|l|}{ Ethnic origin } \\
\hline $\begin{array}{l}\text { Non-Spanish Hispanic } \\
\text { Latino }\end{array}$ & $55576(86.6 \%)$ & $13781(90.7 \%)$ & $<0.0001$ \\
\hline Spanish Hispanic Latino & $8597(13.4 \%)$ & $1420(9.3 \%)$ & \\
\hline \multicolumn{4}{|c|}{ Pathological grading } \\
\hline $\begin{array}{l}\text { Well differentiated, } \\
\text { Grade I }\end{array}$ & $4277(6.7 \%)$ & $682(4.5 \%)$ & $<0.0001$ \\
\hline $\begin{array}{l}\text { Moderately } \\
\text { differentiated, Grade II }\end{array}$ & $14289(22.3 \%)$ & $2614(17.2 \%)$ & \\
\hline $\begin{array}{l}\text { Poorly differentiated, } \\
\text { Grade III }\end{array}$ & $31665(49.3 \%)$ & $5953(39.2 \%)$ & \\
\hline $\begin{array}{l}\text { Undifferentiated, } \\
\text { anaplastic, Grade IV }\end{array}$ & $2244(3.5 \%)$ & $727(4.8 \%)$ & \\
\hline
\end{tabular}

\begin{tabular}{|c|c|c|c|}
\hline Unknown & $11698(18.2 \%)$ & $5225(34.4 \%)$ & \\
\hline \multicolumn{4}{|l|}{ SEER tumor stage } \\
\hline Localized & 21635(33.7\%) & $2028(13.3 \%)$ & $<0.0001$ \\
\hline Distant & $11569(18 \%)$ & $7114(46.8 \%)$ & \\
\hline Regional & 29364(45.8\%) & $2153(14.2 \%)$ & \\
\hline Unstaged & $1605(2.5 \%)$ & $3906(25.7 \%)$ & \\
\hline \multicolumn{4}{|l|}{ Marital status } \\
\hline $\begin{array}{l}\text { Married (including } \\
\text { common law) }\end{array}$ & $41253(64.3 \%)$ & $8287(54.5 \%)$ & $<0.0001$ \\
\hline Separated or divorced & $5383(8.4 \%)$ & $1090(7.2 \%)$ & \\
\hline Single (never married) & 7079(11\%) & 1653(10.9\%) & \\
\hline Widowed & 10458(16.3\%) & $4171(27.4 \%)$ & \\
\hline \multicolumn{4}{|l|}{$\begin{array}{l}\text { Median household } \\
\text { income } \\
(2008-2012) \#\end{array}$} \\
\hline Q1 & 15155(23.6\%) & $4712(31 \%)$ & $<0.0001$ \\
\hline Q2 & $16980(26.5 \%)$ & 2882(19\%) & \\
\hline Q3 & $16805(26.2 \%)$ & $4246(27.9 \%)$ & \\
\hline Q4 & $15233(23.7 \%)$ & $3361(22.1 \%)$ & \\
\hline \multicolumn{4}{|l|}{$\begin{array}{l}\text { high school education } \\
\text { (2008-2012)\# }\end{array}$} \\
\hline Q1 & 15488(24.1\%) & $4473(29.4 \%)$ & $<0.0001$ \\
\hline Q2 & 15885(24.8\%) & $4042(26.6 \%)$ & \\
\hline Q3 & 15212(23.7\%) & $4449(29.3 \%)$ & \\
\hline Q4 & $17588(27.4 \%)$ & $2237(14.7 \%)$ & \\
\hline \multicolumn{4}{|l|}{ CSS status } \\
\hline censored & $28896(45 \%)$ & $2440(16.1 \%)$ & $<0.0001$ \\
\hline dead & $35277(55 \%)$ & $12761(83.9 \%)$ & \\
\hline \multicolumn{4}{|l|}{ OS status } \\
\hline alive & $16585(25.8 \%)$ & $572(3.8 \%)$ & $<0.0001$ \\
\hline dead & $47588(74.2 \%)$ & $14629(96.2 \%)$ & \\
\hline
\end{tabular}

Patients older than 80 years had a higher percentage of noncompliance to surgery, while young patients less than 50 years had the lowest. As to patients' race and origin, we found that non-Spanish Hispanic Latino patients were prone to refuse surgery operation, while more Asian or Pacific Islander patients accepted surgical therapy. With regard to SEER tumor stage, more patients with distant GC were found not to follow surgical therapy compared with the patients with localized GC. Another variable-pathological grading-was not fit for further analysis, considering that a large proportion of patients had no valid information in the surgical noncompliance group (34.4\%). As for the marital status, the widowed patients showed poor compliance of treatment, with the higher likelihood of not following the surgery (27.4\% versus $16.3 \%$ ), while married patients had relatively favorable compliance (64.3\% versus $54.5 \%)$. We have also found that patients from economy and education underdeveloped areas had a larger proportion of nonperforming surgery than other populations.

The constitution ratio of clinical variables was also analyzed by Chi-square test between the surgical noncompliance group and the non-surgical group. As shown in Figure 1A, non-surgical therapeutic regimen has received more attention in recent decades; during the period 1973-1983, no GC patient in this cohort was 
recommended for non-surgical therapy. To the contrary, the proportion of surgical noncompliance decreased gradually over time. Figure $1 \mathrm{~B}$ revealed that the surgical noncompliance group had more elderly patients (older than 65 years) compared with the patients in non-surgical group. Figure 1C\&D showed that more gastric cancers which were at distant stage or poorly differentiated were found in the non-surgical group. Besides the above variables, significant differences can also be seen between the two groups, such as gender, race, origin, marital status, education level and economic income. The detailed results can be found in Table S1.

\section{Identification of factors associated with poor surgical compliance}

In a multivariable logistic regression model,

A

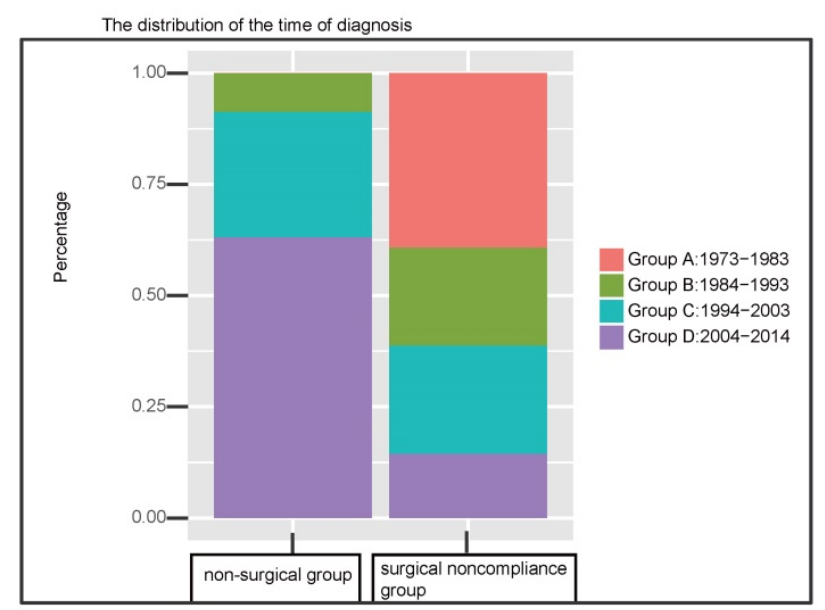

C

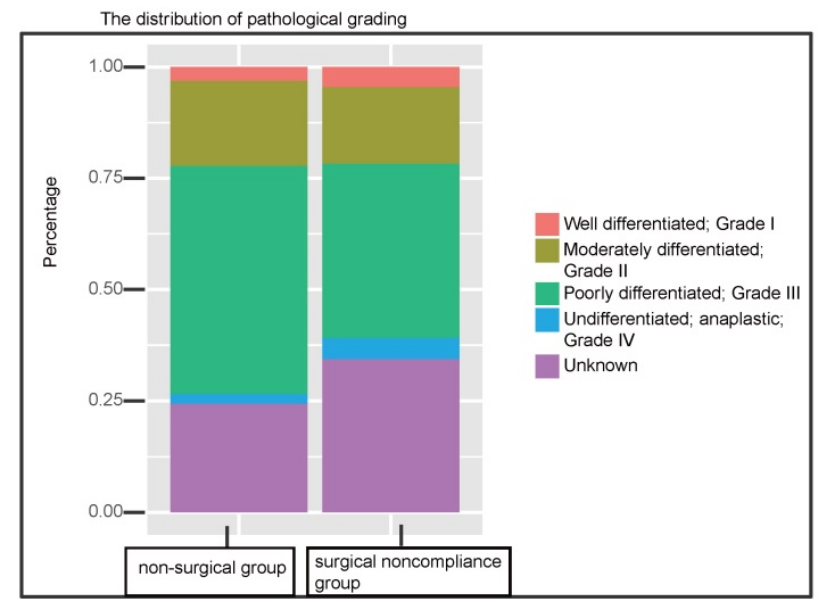

several variables were demonstrated to be significantly associated with poor compliance of surgical treatment when other factors were adjusted for (Figure 2). Patients who were 80 years or older (OR, 3.23; 95\% CI, 2.96-3.35; p < 0.001), American Indian/Alaska Native race (OR, 2.12; 95\% CI, 1.69-2.64; $\mathrm{p}<0.001)$, at distant stage (OR, 5.64; 95\% CI, 5.32-5.99; $\mathrm{p}<0.001)$, single (OR, 1.37; 95\% CI, 1.27-1.47; $\mathrm{p}<0.001$ ), or widowed ( OR, 1.29; 95\% CI,1.22-1.37; $\mathrm{p}<$ 0.001 ) were less likely to follow surgical treatment. The factors associated with accepting surgical therapy included diagnosed recently (GroupD: 2004-2014: OR, $0.21 ; 95 \% \mathrm{CI}, 0.19-0.22 ; \mathrm{p}<0.001)$, Asian or Pacific Islander race (OR, 0.72; 95\% CI, 0.67-0.77; $\mathrm{p}<0.001)$, regional stage (OR, 0.69; 95\% $\mathrm{CI}, 0.64-0.74 ; \mathrm{p}<0.001)$, and higher educational or economic status.

B

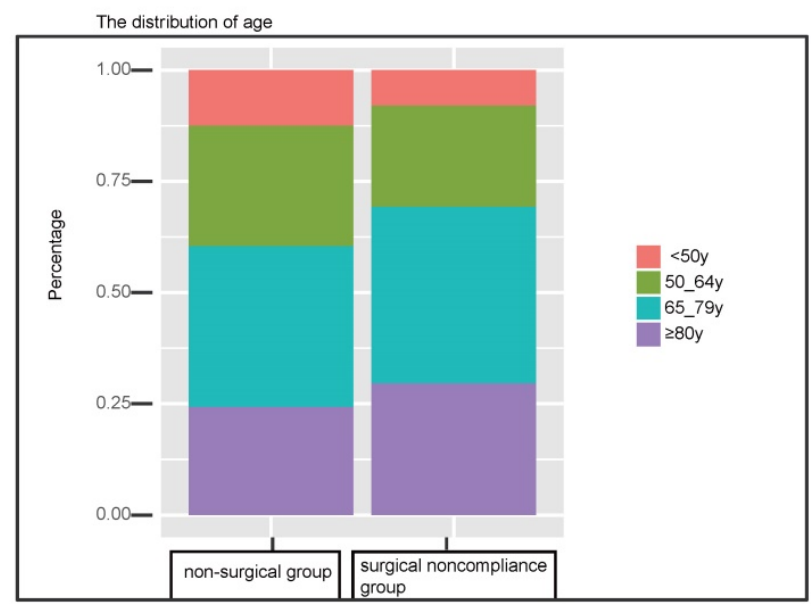

D

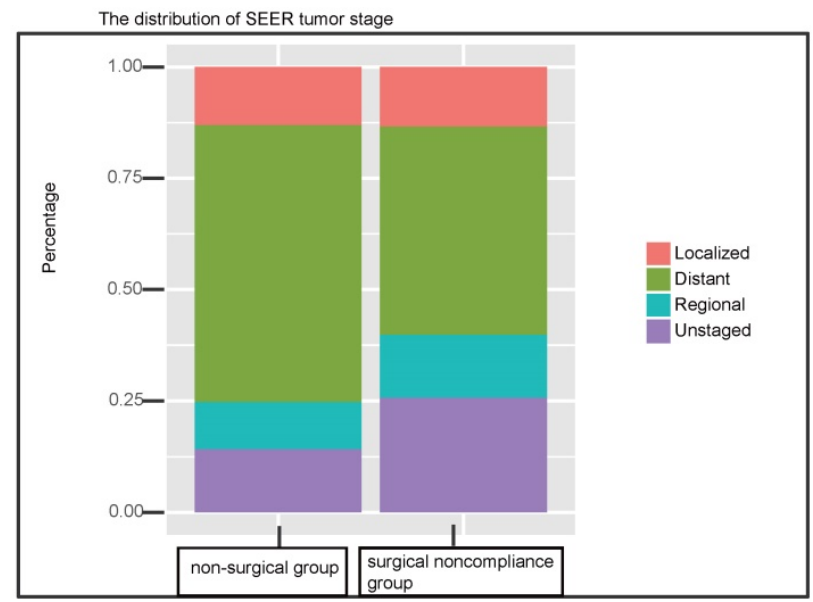

Figure 1: Stacked bars indicate the distribution of variables between surgical noncompliance group and non-surgical group. (A) the distribution of time of diagnosis which is divided into four chronological categories (Group A: 1973-1983, Group B: 1984-1993, Group C: 1994-2003, Group A: 2004-2014). (B) the distribution of age which is factitiously classified into four categories (<50y, 50-64y, 65-79y, $\geq 80 y$ ). (C) the distribution of pathological grading (Well differentiated, Grade I; Moderately differentiated, Grade II; Poorly differentiated, Grade III; Undifferentiated, anaplastic, Grade IV, Unknown). (D) the distribution of SEER tumor stage (Localized; Regional; Distant; Unknown) 


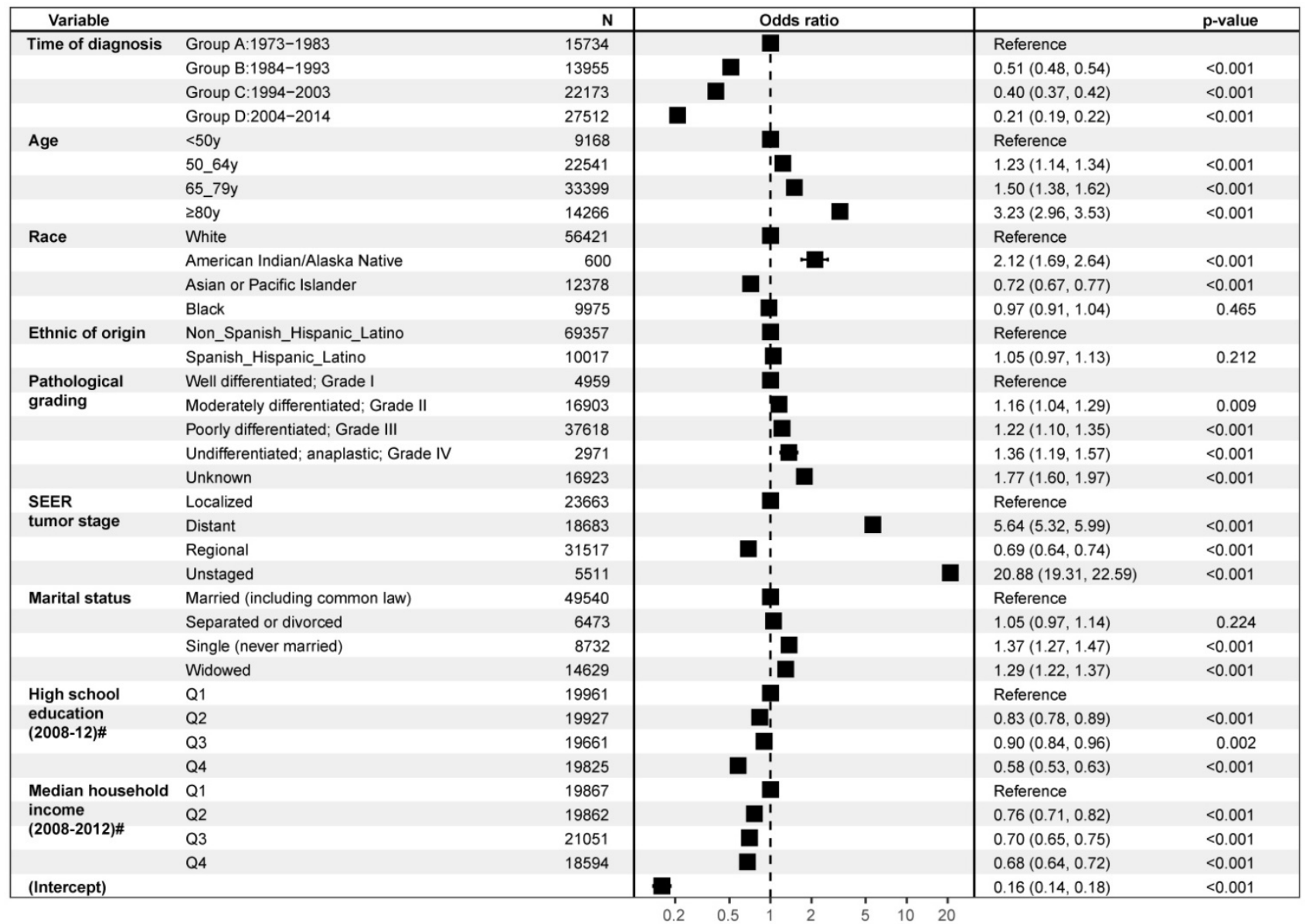

Figure 2: Forest plot of Multivariable Logistic analyses of surgical noncompliance adjusted by the time of diagnosis, gender, age, race, ethnic origin, pathological grading, tumor stage, marital status, education level, economic income. The black squares on the transverse lines represent the hazard ratio (HR), and the transverse lines represent $95 \% \mathrm{Cl}$. \# Measure of educational level or economic income for each patient's area of residence is from 2012 American Community Survey data, and it is categorized into equally proportioned quartiles

A

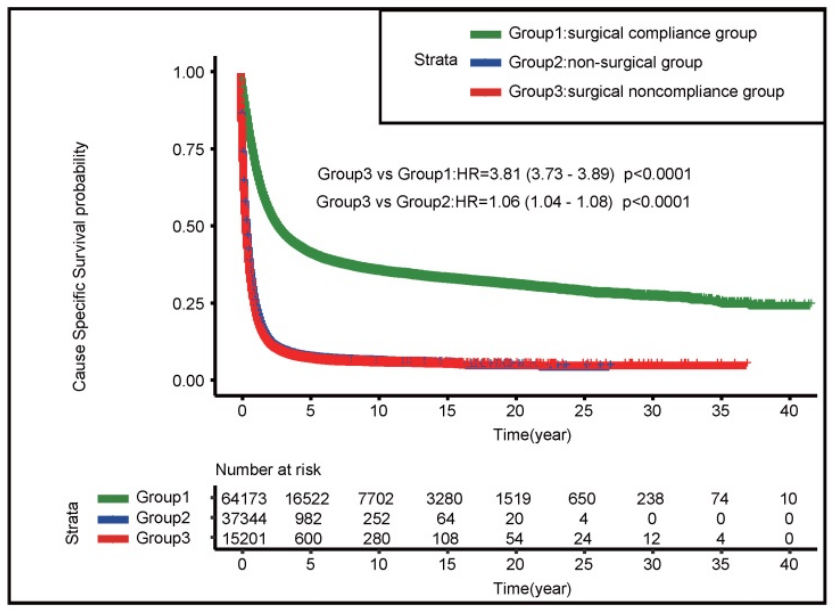

B

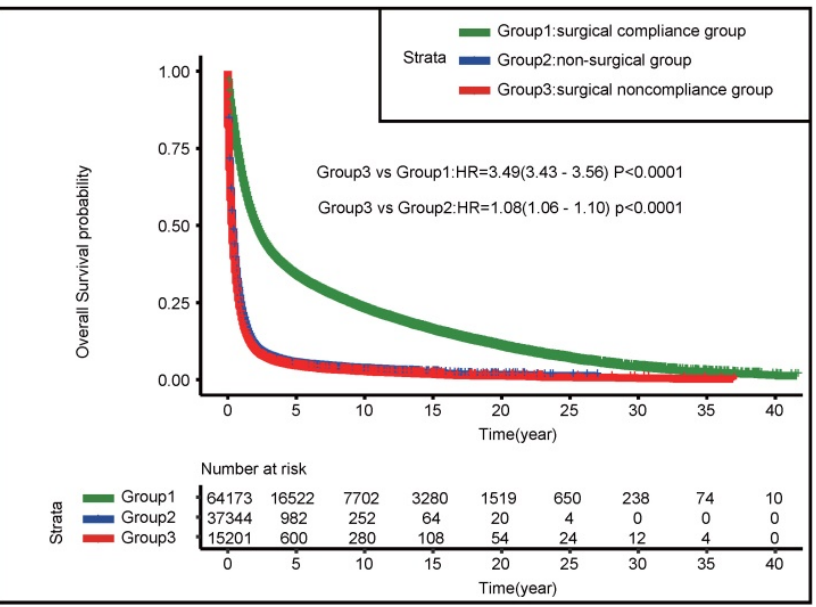

Figure 3: Kaplan-Meier estimates of the Cancer-Specific survival (A) and Overall Survival (B) for the total cohort among three groups (surgical compliance group; surgical noncompliance group; non-surgical group)

\section{Analysis of prognostic factors using Multivariable Cox regression}

In order to investigate which variables can significantly affect GC patients' prognosis.
Multivariable Cox regression analysis was adopted by forcedly entering all the covariates. Supplementary Fig 1 showed the covariates which were significantly associated with patients' CSS when other factors were adjusted for, including time of diagnosis, gender, age, 
race, pathological grading, tumor stage etc. The risk increased when the patients were with old age, poor tissue differentiation and late tumor stage, while the time of diagnosis was a protective factor. Compared with patients diagnosed at 1973-1983, the patients of latest decade owned low risk of cancer-specific death (HR: $0.21,95 \% \mathrm{CI}(0.19,0.22), \mathrm{P}<0.001)$. The results were similar when investigating the risk factors associated with patients' OS (Supplementary Fig 2).

\section{Analysis of the survival outcome}

Kaplan-Meier estimator method showed that the prognosis of GC patients was unfavorable, the five-year OS for the entire cohort was $21.0 \%(95 \% \mathrm{CI}$ : $0.208-0.213$ ) and the five-year CSS was $27.1 \%$ (95\% CI: $0.268-0.274)$.

\subsection{Comparison of the survival outcome between different groups}

The patients were stratified by different surgery status, as shown in Figure 3A, patients in surgical noncompliance group demonstrated worse CSS compared with those in surgical compliance group (HR= 3.81, 95\%CI: 3.73-3.89, $\mathrm{P}<0.0001)$; the results of OS was consistent ( $\mathrm{HR}=3.49,95 \% \mathrm{CI}$ : $3.43-3.56, \mathrm{P}<$ 0.0001) (Figure 3B).

The survival curves of surgical noncompliance group and non-surgical group were almost overlapping, and surgical noncompliance group demonstrated relatively worse outcome compared with non-surgical group. (CSS $\mathrm{HR}=1.06,95 \% \mathrm{CI}$ : 1.04-1.08, $\mathrm{P}<0.0001$; OS $\mathrm{HR}=1.08$, 95\%CI: 1.06-1.1, $\mathrm{P}<0.0001$ ) (Figure $3 \mathrm{~A} \& \mathrm{~B}$ ).

\subsection{Trends in survival stratified by the time of diagnosis}

Multivariable Cox regression indicated that the time of diagnosis was closely associated with patients' survival. In order to investigate the trends in survival, the patients were stratified by the time of diagnosis. Figure 4A showed the results of CSS, although the prognosis gradually improved over time in all three groups, patients in surgical compliance group still presented significantly more favorable survival than the other two groups. During the period 1984-1993, the prognosis in surgical noncompliance group was almost as worse as that in non-surgical group. After the year of 1994, the outcome of surgical noncompliance group turned slightly better than that of non-surgical group, even though both of them remained dismal. The trend of OS was consistent, as shown in Figure 4B.

\section{Discussion}

In our study, we aimed to investigate the effect of surgical compliance on survival among GC patients. We found that nearly $20 \%$ of GC patients did not follow surgical therapy. Although the proportion is declining by years, the health status of these patients shall not be ignored considering the large population base of GC patients. It was unclear whether those patients in surgical noncompliance group have adopted other treatments, such as chemotherapy or radiotherapy, but one thing was very clear that they should have performed surgical therapy according to the therapeutic guidelines. As we have expected, survival analysis demonstrated that the prognosis of surgical noncompliance group was significantly worse than that of surgical compliance group. General speaking, most of the patients in non-surgical group were in advanced stage of tumor or had severe commodities or surgical contraindications, thus the prognosis was relatively poor. Our study indeed found the adverse outcome in non-surgical group. Notably, survival analysis of CSS and OS indicated that the prognosis of surgical noncompliance group came close to that of non-surgical group.

Surgical compliance imposed a great impact on the survival of GC patients, which warranted further investigation to identify the associated demographic and clinicopathological factors. Logistic analysis showed that age was closely related to the compliance of surgery. The risk increased with age, especially when the patients were older than 80 years. These patients were more than three times likely not to accept surgery. Elderly patients were always with poor functional reserves and more preoperative comorbidities, which increased the risks of surgery. The therapeutic regimen for old ones was relatively conservative, for example, gastrectomy with reduced nodal dissection was an alternative [20, 21]. Inaccurate assessment of surgical risks and benefits for elderly GC patients may be related to poor surgical compliance. Up to now, there was still contradictory evidence related to the postoperative morbidity, mortality, and complications for gastrectomy in old patients. Some investigations found that GC surgery achieved reasonable long-term survival, however, the risk of severe complications increased [18, 22]. On the contrary, Mikami K et al. [20] found that standard gastrectomy in elderly GC patients was with a lower proportion of complications compared to gastrectomy with reduced nodal dissection. Other studies also demonstrated that although the frequency of preoperative comorbidities was significantly high in elderly GC patients compared with young patients, no significant difference of the postoperative complication rate was found between them $[16,17$, $23]$, indicating it was feasible to perform gastrectomy in elderly patients. 
A

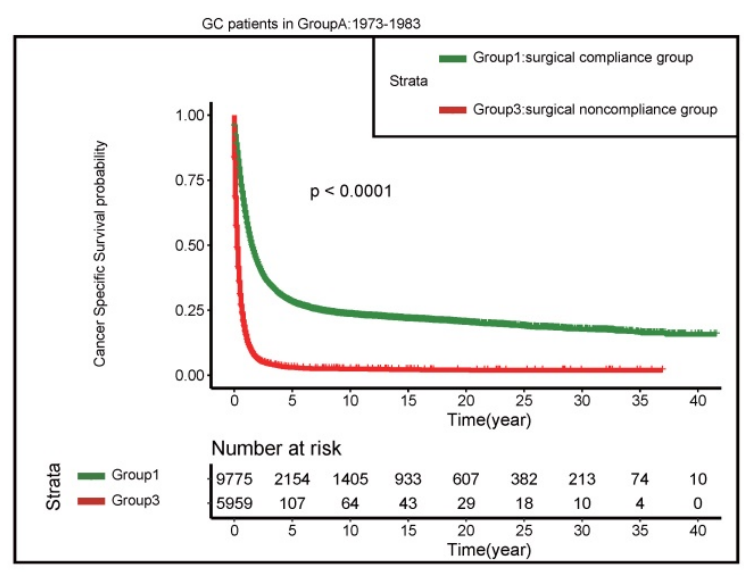

GC patients in GroupB:1984-1993

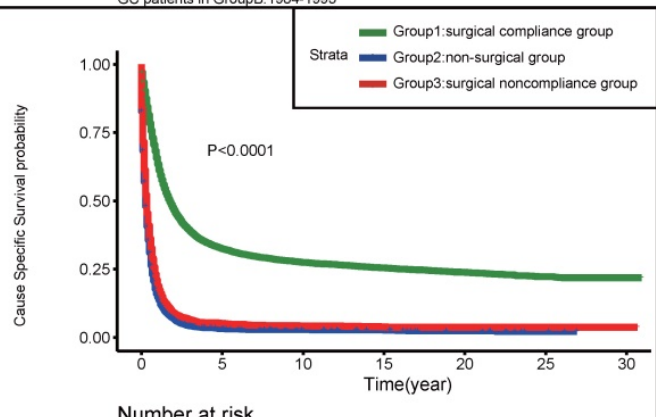

- Group1 Number at risk

焉 $=$ Group2
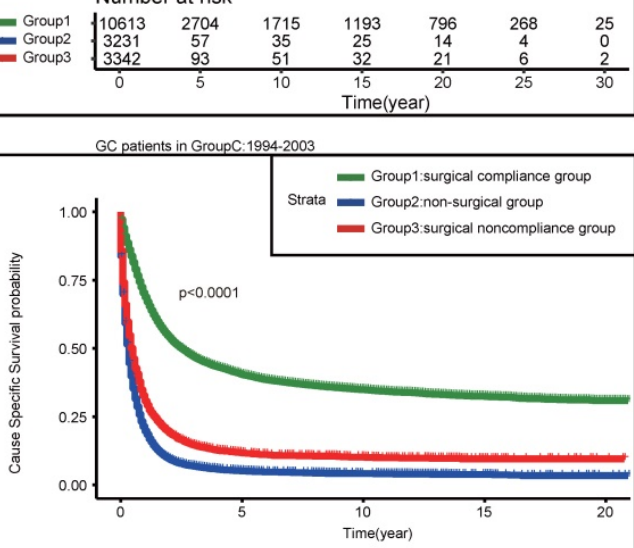

Number at risk

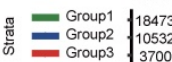

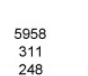

3966
172

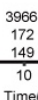

Time(ye

GC patients in GroupD:2004-2014

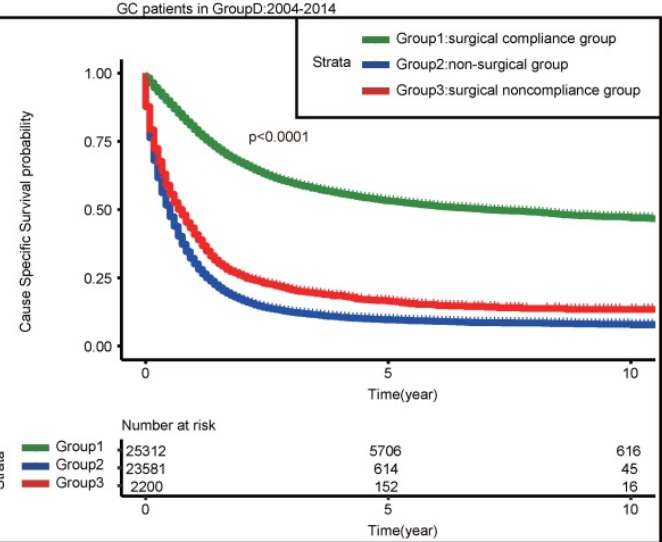

B
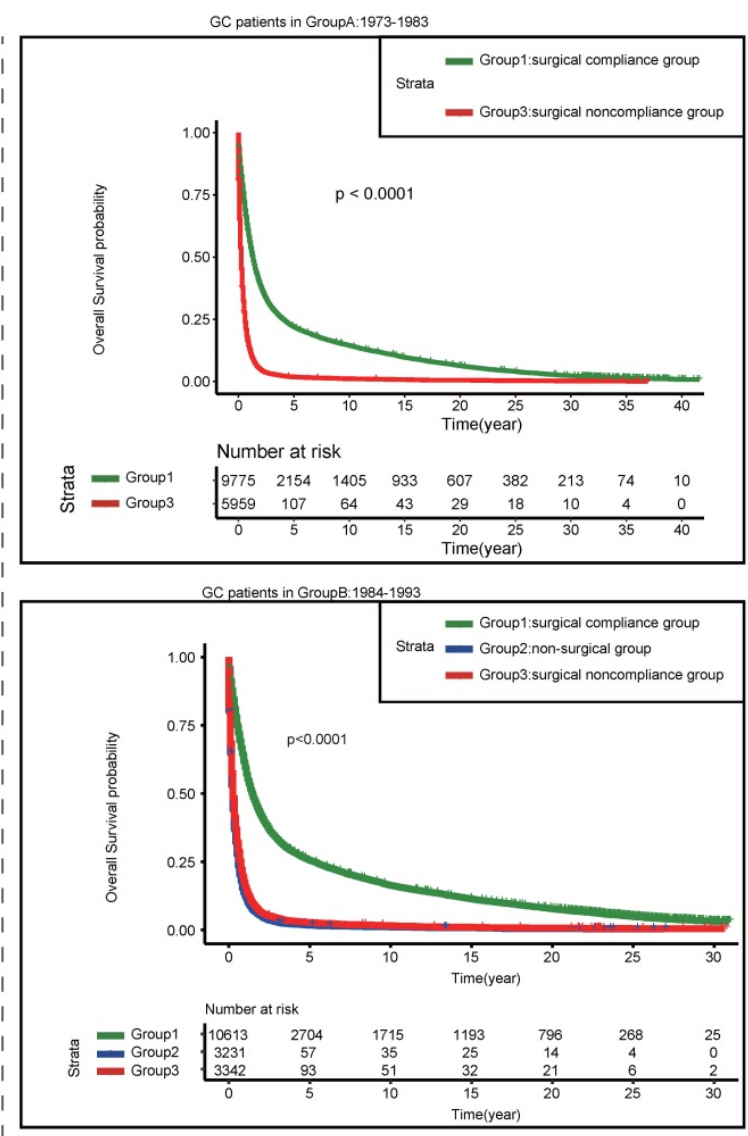

GC patients in GroupC:1994-2003

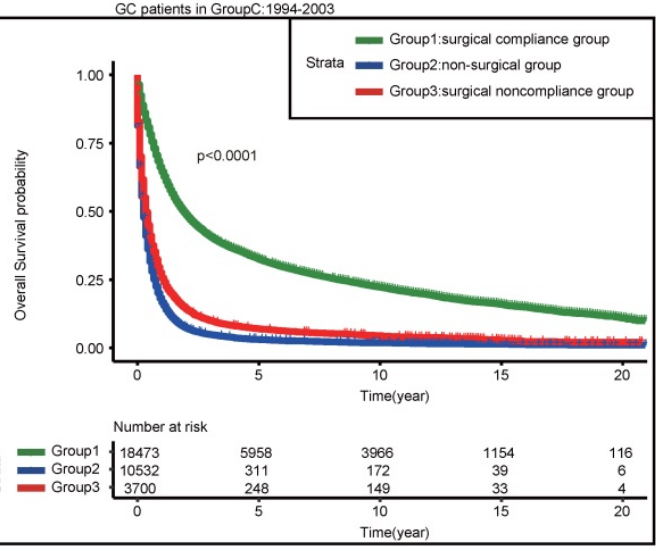

GC patients in GroupD:2004-2014

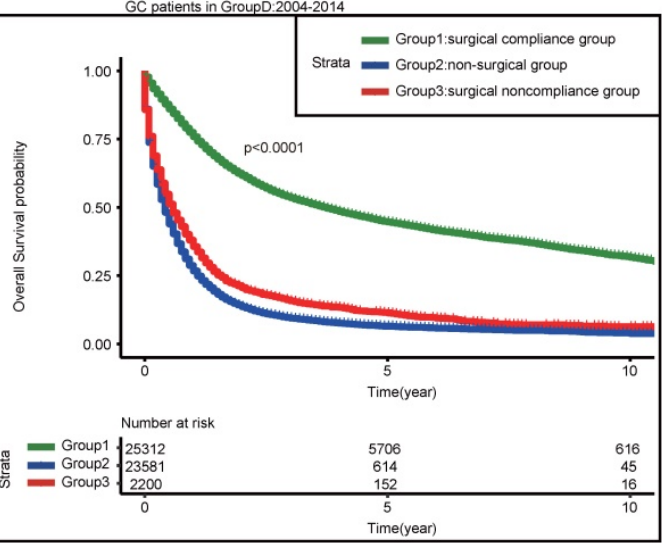

Figure 4: Kaplan-Meier estimates of the Cancer-Specific survival (A) and Overall Survival (B) for the patients diagnosed in different period of time among three groups (surgical compliance group; surgical noncompliance group; non-surgical group) 
The disparity of postoperative complications was probably related to the discrepant preoperative/postoperative management and surgical quality gaps. Well cancer management was the important prerequisite of the surgical treatment for elderly GC patients, which can contribute to build up confidence for clinical practitioners and patients, thus improve compliance with surgery.

Studies indicated that treatment selection in cancer was discrepant among ethnic group[24], and race can be considered to be a predictor of treatment and prognosis for patients with resectable GC [25]. Our study demonstrated that race was closely associated with surgical compliance. Being American Indian/Alaska Native (AI/ANs) increased the likelihood of noncompliance with surgery, while Asian/Pacific Islander showed well compliance. AI/ANs had higher mortality than non-Hispanic white (NHW) with some cancers, and a large proportion of $\mathrm{AI} / \mathrm{ANs}$ cancer patients were with multiple-comorbidities conditions [26]. Moreover, $\mathrm{AI} / \mathrm{ANs}$ were observed to start cancer therapy later than NHWs [27]. A study revealed some barriers to access cancer treatment for $\mathrm{AI} / \mathrm{AN}$ breast cancer patients, such as lack of cancer care at local clinics, and long time waiting in the clinic for cancer care etc[28], which might account for the poor compliance to surgery for AI/ANs. The incidence of GC was high in Asians/Pacific Islanders in the United States [29], Asian GC patients were diagnosed at a younger age and had superior overall survival [30]. High risk of GC in Asia-Pacific region made the screening and prevention system better [31], so it can be speculated that increased awareness to GC for Asian or Pacific Islander patients might contribute to treatment compliance. Liu et al. [32] found that the black were more likely to not receive treatment in elderly GC patients, but we had not found a significant difference between the black and the white patients as to surgical compliance in our study. The study of Liu et al. focused only on the elder GC patients and ours had no limitation on age [32], so the disparity in research group might contribute to this discrepancy.

The effect of socioeconomic status on tumor was taken more and more seriously. One study demonstrated that the likelihood of receiving radiotherapy and the survival rates were significantly affected by education level and economic income etc; patients with well socioeconomic status were prone to receipt radiotherapy [25]. This was consistent with our analysis; the likelihood of noncompliance with surgery was inversely proportional to the level of education and economic income. Education level was associated with the understanding of disease, and good economic condition was the guarantee of active treatment, probably both of which impacted the selection of and compliance with therapeutic regimen together. The relation between marital status and the tumor has been widely analyzed. Marital status imposed a significant influence on patients' survival, and the risk of cancer mortality was greater for unmarried patients [33, 34]. In our analysis, the married patients were inclined to receipt surgery compared to the unmarried ones, so the better prognosis for married patients might be partly attributable to the well surgical compliance.

Besides demographic factors, clinical factors were also analyzed through logistic regression, although such variables were generally considered to be indirectly related to patients' compliance. We found that tumor stage was also closely associated with surgical noncompliance in GC patients; patients who were in the advanced stage of tumor were less likely to accept surgery. Patients with advanced cancer always had adverse outcome [35], which perhaps deprived the treatment confidence of patients and resulted in poor compliance to surgery in the end. As for cancers, early detection and early treatment were pivotal for beneficial outcomes. Interestingly, our study demonstrated that the patients with regional GC were more likely to receipt surgical therapy compared with the patients with localized GC, indicated that there were not adequate and timely treatments for these GC patients who were diagnosed at a relatively early stage. The reasons for relative inadequate surgical compliance for such patients were unknown; it is probably due to lack of knowledge of disease. This finding warrants further investigation.

The reasons why those patients are not recommended for surgical treatment can be roughly divided into the following two categories. One is that patients cannot tolerate surgical treatment because of severe comorbidities; the other is that one has lost the opportunity of surgical treatment as cancer has extensively metastasized. Because of poor health condition and lack of effective treatment, the patients of non-surgical group showed significant adverse outcome. As for the patients in surgical noncompliance group, the physical and performance status shall be better than the patients in non-surgical group, thus they were expected to have a more favorable prognosis. Surprising, the outcome of surgical noncompliance group was almost as bad as non-surgical group.

Cox regression analysis was performed to identify the associated prognostic factors, and we found that the GC patients with elderly age, poor tissue differentiation, advanced stage of tumor, the early decade of diagnosis time or non-surgical treatment had a high risk of adverse outcome. 
The age of diagnosis impacted nodal metastases and survival in GC, and the outcome became worse with age [36]. Our analysis yielded the consistent results; the ratio of elderly patients to young ones was significantly larger in surgical noncompliance group than that in surgical compliance group. Importantly, the outcome of GC patients was also closely associated with tumor grading and tumor staging. Compared with non-surgical group, patients in surgical noncompliance group shall have a relatively better health condition and favorable survival; as expected, the proportion of patients with poorly differentiated and/or advanced GC was less in surgical noncompliance group. However, the actual outcome of GC patient in surgical noncompliance group was not strictly what we have expected. When stratified by the time of diagnosis, we found that the outcome of surgical noncompliance group was slightly better than that of non-surgical group after the year of 1994, but both of them were still very poor.

These findings indicated that the disparities in treatment compliance might be a pivotal factor that affected the prognosis of GC patients. Because of the high risk of distant metastasis and local recurrence after gastrectomy, systemic therapy was mandatory for most of GC patients. The extensive treatments included surgery, adjuvant therapy, neoadjuvant therapy, targeted therapy or immunotherapy, either alone or in combinations [37]. Any of the treatment modalities took the good therapy compliance as the premise. The poor compliance to surgery probably indicated poor compliance with other treatment regimens, thus resulted in adverse outcome at last.

There still are some limitations to be acknowledged in our study. Firstly, some important confounding factors associated with surgical compliance are not available in the SEER database, such as patients' performance status, dietary patterns, obesity, religion etc. Secondly, some subgroups in our study are small size, such as patients in the American Indian/Alaskan subgroup, which might preclude us to carry out reliable analysis. Thirdly, considering the potential statistic error caused by the small sample size, the data spanned a long time is used; then another problem will be hard to avoid, as the incidence, treatment modality and outcome in cancer changes over time, it may not exactly mirror the current situation of treatment for GC patients.

\section{Conclusion}

The SEER database was used to evaluate the survival disparity of GC patients based on surgical compliance. GC patients with good compliance to surgery had better survival, while the outcome of noncompliance patients was comparable to that of non-surgical patients. The poor surgical compliance for GC patients were related to older age, American Indian/Alaska Native race, poor tissue differentiation/advanced stage of tumor, single/widowed status, lower socioeconomic status and earlier time of diagnosis.

\section{Abbreviations}

CI: confidence interval; CSS: cause-specific survival; GC: gastric cancer; HR: hazard ratio; OS: overall survival; SEER: Surveillance Epidemiology and End Results databases

\section{Supplementary Material}

Supplementary figures and tables. http://www.jcancer.org/v10p0779s1.pdf

\section{Acknowledgement}

This project was supported by the National Natural Science Foundation of China (Grant No: 81602558).

\section{Competing Interests}

The authors have declared that no competing interest exists.

\section{References}

1. Ferlay J, Soerjomataram I, Dikshit R, et al. Cancer incidence and mortality worldwide: sources, methods and major patterns in GLOBOCAN 2012. Int J Cancer. 2015; 136: E359-86.

2. Ferlay J, Steliarova-Foucher E, Lortet-Tieulent J, et al. Cancer incidence and mortality patterns in Europe: estimates for 40 countries in 2012. Eur J Cancer. 2013; 49: 1374-403.

3. Jemal A, Bray F, Center MM, et al. Global cancer statistics. CA Cancer J Clin. 2011; 61: 69-90.

4. Siegel RL, Miller KD, Jemal A. Cancer statistics, 2018. CA Cancer J Clin. 2018; 68: 7-30.

5. Ferro A, Peleteiro B, Malvezzi M, et al. Worldwide trends in gastric cancer mortality (1980-2011), with predictions to 2015, and incidence by subtype. Eur J Cancer. 2014; 50: 1330-44.

6. Van Cutsem E, Dicato M, Geva R, et al. The diagnosis and management of gastric cancer: expert discussion and recommendations from the 12th ESMO/World Congress on Gastrointestinal Cancer, Barcelona, 2010. Ann Oncol. 2011; 22 Suppl 5: v1-9.

7. Lutz MP, Zalcberg JR, Ducreux M, et al. Highlights of the EORTC St. Gallen International Expert Consensus on the primary therapy of gastric, gastroesophageal and oesophageal cancer - differential treatment strategies for subtypes of early gastroesophageal cancer. Eur J Cancer. 2012; 48: 2941-53.

8. Choi MK, Kim GH, Park DY, et al. Long-term outcomes of endoscopic submucosal dissection for early gastric cancer: a single-center experience. Surg Endosc. 2013; 27: 4250-8.

9. Cai J, Wei D, Gao CF, et al. A prospective randomized study comparing open versus laparoscopy-assisted D2 radical gastrectomy in advanced gastric cancer. Dig Surg. 2011; 28: 331-7.

10. Schwarz RE, Zagala-Nevarez K. Recurrence patterns after radical gastrectomy for gastric cancer: prognostic factors and implications for postoperative adjuvant therapy. Ann Surg Oncol. 2002; 9: 394-400.

11. Ko KJ, Shim JH, Yoo HM, et al. The clinical value of non-curative resection followed by chemotherapy for incurable gastric cancer. World J Surg. 2012; 36: 1800-5.

12. Hallissey MT, Dunn JA, Ward LC, et al. The second British Stomach Cancer Group trial of adjuvant radiotherapy or chemotherapy in resectable gastric cancer: five-year follow-up. Lancet. 1994; 343: 1309-12.

13. Dassen AE, Lemmens VE, van de Poll-Franse LV, et al. Trends in incidence, treatment and survival of gastric adenocarcinoma between 1990 and 2007: a population-based study in the Netherlands. Eur J Cancer. 2010; 46: 1101-10.

14. Verdecchia A, Francisci S, Brenner H, et al. Recent cancer survival in Europe: a 2000-02 period analysis of EUROCARE-4 data. Lancet Oncol. 2007; 8: 784-96.

15. Orditura M, Galizia G, Sforza V, et al. Treatment of gastric cancer. World J Gastroenterol. 2014; 20: 1635-49. 
16. Orsenigo E, Tomajer V, Palo SD, et al. Impact of age on postoperative outcomes in 1118 gastric cancer patients undergoing surgical treatment. Gastric Cancer. 2007; 10: 39-44.

17. Sakurai K, Muguruma K, Nagahara H, et al. The outcome of surgical treatment for elderly patients with gastric carcinoma. J Surg Oncol. 2015; 111: 848-54.

18. Fujiwara $\mathrm{Y}$, Tsujie M, Hara J, et al. Comparison of gastric cancer surgery between patients aged $>80$ years and $<79$ years: complications and multivariate analysis of prognostic factors. Hepatogastroenterology. 2014; 61: 1785-93.

19. Hundahl SA, Macdonald JS, Benedetti J, et al. Surgical treatment variation in a prospective, randomized trial of chemoradiotherapy in gastric cancer: the effect of undertreatment. Ann Surg Oncol. 2002; 9: 278-86.

20. Mikami K, Hirano K, Futami K, et al. Gastrectomy with limited surgery for elderly patients with gastric cancer. Asian J Surg. 2018; 41: 65-72.

21. Evers BM, Townsend CM, Jr., Thompson JC. Organ physiology of aging. Surg Clin North Am. 1994; 74: 23-39.

22. Yang JY, Lee HJ, Kim TH, et al. Short- and Long-Term Outcomes After Gastrectomy in Elderly Gastric Cancer Patients. Ann Surg Oncol. 2017; 24: 469-477.

23. Hikage $M$, Tokunaga $M$, Makuuchi $R$, et al. Surgical outcomes after gastrectomy in very elderly patients with gastric cancer. Surg Today. 2018

24. Campbell DE, Greenberg ER. Racial differences in the treatment of early-stage lung cancer. N Engl J Med. 2000; 342: 517; author reply 518-9.

25. Stessin AM, Sherr DL. Demographic disparities in patterns of care and survival outcomes for patients with resected gastric adenocarcinoma. Cancer Epidemiol Biomarkers Prev. 2011; 20: 223-33.

26. Emerson MA, Banegas MP, Chawla N, et al. Disparities in Prostate, Lung, Breast, and Colorectal Cancer Survival and Comorbidity Status among Urban American Indians and Alaskan Natives. Cancer Res. 2017; 77: 6770-6776.

27. Adams SV, Bansal A, Burnett-Hartman AN, et al. Cancer Treatment Delays in American Indians and Alaska Natives Enrolled in Medicare. J Health Care Poor Underserved. 2017; 28: 350-361.

28. Goodwin EA, Burhansstipanov L, Dignan M, et al. The experience of treatment barriers and their influence on quality of life in American Indian/Alaska Native breast cancer survivors. Cancer. 2017; 123: 861-868.

29. Brown LM, Devesa SS. Epidemiologic trends in esophageal and gastric cancer in the United States. Surg Oncol Clin N Am. 2002; 11: 235-56.

30. Chen Y, Haveman JW, Apostolou C, et al. Asian gastric cancer patients show superior survival: the experiences of a single Australian center. Gastric Cancer. 2015; 18: 256-61.

31. Fock KM, Talley N, Moayyedi $\mathrm{P}$, et al. Asia-Pacific consensus guidelines on gastric cancer prevention. J Gastroenterol Hepatol. 2008; 23: 351-65.

32. Liu N, Molena D, Stem M, et al. Underutilization of Treatment for Regional Gastric Cancer Among the Elderly in the USA. J Gastrointest Surg. 2018.

33. Qiu M, Yang D, Xu R. Impact of marital status on survival of gastric adenocarcinoma patients: Results from the Surveillance Epidemiology and End Results (SEER) Database. Sci Rep. 2016; 6: 21098.

34. Jin JJ, Wang W, Dai FX, et al. Marital status and survival in patients with gastric cancer. Cancer Med. 2016; 5: 1821-9.

35. Marano L, Boccardi V, Braccio B, et al. Comparison of the 6th and 7th editions of the AJCC/UICC TNM staging system for gastric cancer focusing on the "N" parameter-related survival: the monoinstitutional NodUs Italian study. World J Surg Oncol. 2015; 13: 215.

36. Ahmad A, Khan H, Cholankeril G, et al. The impact of age on nodal metastases and survival in gastric cancer. J Surg Res. 2016; 202: 428-35.

37. Van Cutsem E, Sagaert X, Topal B, et al. Gastric cancer. Lancet. 2016; 388: 2654-2664. 